\title{
Germanica
}

\section{„Daß man gestorben sein muß, um ganz ein Schaffender zu sein"}

Der Tod in Thomas Manns frühen Erzählungen

"That one must have died in order to be altogether a creator." Death in Thomas Mann's early works "Qu'il faille être mort pour être tout à fait un créateur ». La mort dans les récits de jeunesse de Thomas Mann

\section{Frédéric Weinmann}

\section{(2penEdition}

\section{Journals}

Édition électronique

URL : http://journals.openedition.org/germanica/3734

DOI : ERREUR PDO dans /localdata/www-bin/Core/Core/Db/Db.class.php L.34 : SQLSTATE[HY000]

[2006] MySQL server has gone away

ISSN : 2107-0784

Éditeur

Université de Lille

Édition imprimée

Date de publication : 30 juin 2017

Pagination : 103-116

ISBN : 9782913857391

ISSN : 0984-2632

Référence électronique

Frédéric Weinmann, „m,Daß man gestorben sein muß, um ganz ein Schaffender zu sein “" Germanica [Online], 60 | 2017, Online erschienen am: 30 Juni 2019, abgerufen am 08 Januar 2021. URL: http:// journals.openedition.org/germanica/3734 ; DOI: https://doi.org/10.4000/germanica.3734 


\title{
„Daß man gestorben sein muß, um ganz ein Schaffender zu sein.“ Der Tod in Thomas Manns frühen Erzählungen
}

\author{
Frédéric WEINMANN \\ Lycée Hélène Boucher, Paris
}

In Thomas Manns allerersten, oft aufeinander paarweise antwortenden Erzählungen spielt der Tod eine auffallend große Rolle: Beinahe die Hälfte der zwischen 1893 und 1903 veröffentlichten Kurzgeschichten ${ }^{1}$ enden mit dem eintretenden oder zumindest bevorstehenden Ableben des Protagonisten. Außerdem stirbt in Tobias Mindernickel und in Tristan zwar nicht die männliche Hauptfigur, aber immerhin deren Opfer, ob ein gesunder Hund oder eine kranke Frau. Einer dieser Erzählungen gibt eines der Leitmotive in Manns Jugendwerk sogar ihren Titel: Der Tod besteht aus den Aufzeichnungen eines Grafen, der überzeugt ist, am 12. Oktober seines vierzigsten Lebensjahres sterben zu müssen, und vom 10. September an das Tagebuch seiner vermeintlich letzten Wochen und Tage auf Erden führt. Die keineswegs fantastische, sondern beinahe kafkaeske Geschichte handelt eigentlich von einem erweiterten Selbstmord, da die zwölfjährige Tochter des Ich-Erzählers und einer, inzwischen verstorbenen, Portugiesin am 11. Oktober um 23 Uhr einem Herzinfarkt erliegt. Ob der Vater um Mitternacht auch tatsächlich dahin-

1. - Gemeint sind: Enttäuschung (1896), Der Wille zum Glück (1896), Der Tod (1897), Der kleine Herr Friedemann (1897), Luischen (1900) und Der Weg zum Friedhof (1900). 
geht, bleibt anzunehmen: Die Notizen enden mit Auslassungspunkten vor dem letzten Atemzug des Schreibenden.

Man mag sich fragen, warum ein vom Leben eher begünstigter Zwanzigjähriger mit solcher Besessenheit das leidige Thema immer wieder aufs Tapet bringt. Am Ende seines Beitrags ,,Zu einem Porträt Thomas Manns" erklärte Theodor Adorno 1962 diese morbide Anziehung nicht wie sonst üblich durch eine dekadente, etwa von Detlev Spinell in Tristan verkörperte Gemütslage, sondern im Gegenteil als ein Spiel mit dem Unabwendbaren, als eine Form der Anerkennung gegenüber der Kraft, der Macht der Natur. So schreibt er:

Wenn seine Schriften ihre Mitte im Tod zu haben scheinen, so ist daran kaum die Todessehnsucht schuld, kaum auch nur besondere Affinität zum Verfall, sondern insgeheim List und Aberglaube: das stets Angerufene und Beredete eben dadurch fortzuhalten und zu bannen. Dem Tod, dem blinden Naturzusammenhang hat sein Ingenium widerstanden wie sein Körper. Die Manen des Dichters mögen es mir verzeihen, aber er war kerngesund. [...] Was sein Werk veranlasste, die Komplizität mit dem Tod zu betonen, die man ihm gar zu gern geglaubt hat, war am Ende wohl etwas von der Ahnung der Schuld darin, dass man überhaupt ist, gleichsam ein Anderes, Mögliches um die eigene Wirklichkeit bringt, indem man seinen Platz einnimmt; er brauchte nicht erst Schopenhauer, um das zu erfahren. Wollte er den Tod überlisten, so hielt er zugleich Kompanie mit ihm aus dem Gefühl, dass es keine Versöhnung des Lebendigen gibt als Ergebung: nicht Resignation ${ }^{2}$.

Gewiss soll Skepsis gegenüber einer leichtfertigen Übertragung der Todessehnsucht männlicher Protogonisten - so zahlreich sie auch sein mögen - auf die Person des Schriftstellers schon aus Prinzip geboten sein: Thomas Mann ist nicht Detlev Spinell oder Tonio Kröger. Zu Recht warnt der große Mann-Kenner Hans Rudolf Vaget vor der Neigung, „die biographische Wahrheit aus den Dichtungen zu destillieren“, bzw. vor der „Rückübersetzung“ von Gedichtetem „,ins Gelebte“, die die „bewundernswerte Biographie von Hermann Kurzke“3 zum Beispiel aller Verdienste zum Trotz unweigerlich als fragwürdiges Unterfangen erscheinen lässt. Die grundsätzliche Unterscheidung zwischen realexistierendem Autor und Erzähler oder anderen Romanfiguren erscheint

2. - Theodor W. Adorno, „Zu einem Porträt Thomas Manns“, in: Ders., Gesammelte Schriften, hrsg. von Rolf Tiedemann, Frankfurt a.M., Suhrkamp, 1974, Bd. 11, S. 335-344.

3. - Siehe Hans Rudolf Vaget, ,"Vom höheren Abschreiben”. Thomas Mann, der Erzähler", in: Thomas Sprecher (Hrsg.), Liebe und Tod - in Venedig und anderswo, Frankfurt a.M., Vittorio Klostermann (Thomas-Mann-Studien 33), 2005, S. 15-31, hier S. 22. 
aber umso notwendiger, als dass Thomas Mann, wie die neuerliche Forschung und nicht zuletzt die Tagebücher des Nobelpreisträgers zur Genüge gezeigt haben, als Meister der Verstellung gelten darf.

Der noch junge Schriftsteller gestand offenherzig in einem Brief vom 21. Juli 1897 an seinen Jugendfreund Otto Grautoff, er habe seit dem Kleinen Herrn Friedemann ,diskrete Formen und Masken“4 gefunden, um seine Gefühle zum Ausdruck zu bringen. Wie die Vermummung seiner intimsten Regungen im Frühwerk (d.h. bis zum Tod in Venedig) tatsächlich aussieht, hat inzwischen Kristian Larsson in seiner Masken des Erzählens betitelten Dissertation ${ }^{5}$ ausführlich dargestellt: Ausgehend von Dorrit Cohns Analyse der erzählerischen Unzuverlässigkeit im Tod in Venedig nennt Larsson vier Verfahrensweisen (narrative Inkompetenz, Pseudo-Auktorialität, simulierte Fokalisierung und Verschlüsselung von Sinnzusammenhängen), durch die Mann seine eigentliche Aussage verschlüsselt. Dabei bezieht sich Larsson wie die meisten Biographen und Philologen vorwiegend auf das „offene Geheimnis“ 6 der homoerotischen Veranlagung, die ein Lebensgefühl des Außenseitertums und der Fremdheit hervorruft. In diesem Zusammenhang ist von Manns Todeserotik die Rede.

So vertritt zum Beispiel der oben genannte Biograph Kurzke die Meinung, seit dem Friedemann sei „,sein Stil im wesentlichen fertig“, und behauptet in Anlehnung an Erika Mann, ,dass alle seine Liebesgeschichten dem Bereich des Verbotenen und Tödlichen angehörten“7. Der Tod reduziert sich somit auf eine Chiffre für die unmögliche Liebe im wirklichen Leben. Er wird lediglich auf der symbolischen Ebene in Betracht gezogen, als eine Maske, hinter der sich eine unterschwellige Botschaft verberge. Dabei vergisst man allerdings, dass der junge Schriftsteller sehr wohl eine traumatisierende Erfahrung mit dem Tod zu verarbeiten hatte, nämlich den relativ überraschenden Tod des Vaters.

Paul Thomas Mann war gerade sechzehneinhalb Jahre alt, als Senator Thomas Johann Heinrich Mann am 13. Oktober 1891 infolge einer wahrscheinlich im Juli vorgenommenen chirurgischen Operation an

4. - Thomas Mann, Briefe I, 1889-1913, hrsg. von Thomas Sprecher, Hans R. Vaget, Cornelia Bernini, Frankfurt a. M., Fischer (Große kommentierte Frankfurter Ausgabe, Bd. 21), 2001, S. 87-90, hier S. 89.

5. - Kristian Larsson, Masken des Erzählens. Studien zur Theorie narrativer Unzuverlässigkeit und ihrer Praxis im Frühwerk Thomas Manns, Würzburg, Königshausen \& Neumann (Epistemata, Reihe Literaturwissenschaft 718), 2011.

6. - Siehe Heinrich Detering, Das offene Geheimnis. Zur literarischen Produktivität eines Tabus von Winckelmann bis zu Thomas Mann, Göttingen, Wallstein, 2002.

7. - Hermann Kurzke, Thomas Mann. Das Leben als Kunstwerk. Eine Biographie, Frankfurt a.M., Fischer (Taschenbuch), 2001 (1999), S. 86. 
Pyämie bzw. Sepsis starb ${ }^{8}$. Der Eingriff hatte - vermutlich aus Gründen der Respektabilität - nicht im Lübecker Allgemeinen Krankenhaus, sondern im Familienhaus bzw. im großen Ballsaal der prachtvollen Villa in der Beckergrube stattgefunden. Danach zog sich der einundfünfzigjährige Senator ,zur Kräftigung auf einige Wochen nach Travenmüde“9 zurück, bevor er brutal verschied. Die fünf Kinder oder zumindest die vier älteren (wenn man von dem einjährigen Viktor absieht) wohnten also aus direkter Nähe dem gesamten Drama bei. 1919 gedenkt Thomas immer noch ,dem totkranken Papa, der auf den Treppenabsatz herausgekommen war" 10 . Viel später noch sagte außerdem der nun betagte Nobelpreisträger in einer Ansprache in Lübeck: ,[I Ich] habe nie die umfassende Trauer vergessen, mit der, als ich fünfzehn Jahre alt war, seine Stadt, die ganze Stadt, ihn zu Grabe brachte. Ich kann wohl sagen: sein Bild hat immer im Hintergrunde gestanden alles meines Tuns $[\ldots]$ ". 11 .

Der Schock der unmittelbaren Konfrontation mit Krankheit und Tod für den Halberwachsenen (er selber hätte „Knaben“ geschrieben) wurde durch die ebenso schnellen Folgen des unerwarteten Hingangs des Familienoberhauptes verstärkt: „Die Bewusstseinseinschärfung hatte [...] eingesetzt“", bemerkt Kurzke, ,als mit dem Tod des Vaters, dem Verkauf der Firma und dem Wegzug der Mutter ein rasanter sozialer Abstieg erfolgt war."12 Es wundert daher umso mehr, dass der einfühlsame Biograph die Bedeutung der endgültigen Trennung mit dem Vater für den sensiblen Jungen sozusagen auf den sozialen Aspekt reduziert, wenn er schreibt: „Wäre der Vater am Leben geblieben, hätte der Sohn als Mitglied der Lübecker Aristokratie niemals einen Roman wie Buddenbrooks schreiben können, der so viele Interna aus der eigenen und anderen ersten Familien der Stadt preisgab."13 Ohne hier auf die psychologischen Konsequenzen der somit unmöglich gewordenen Selbstbehauptung durch in der Adoleszenz identitätsstiftende Auflehnung gegen die Vaterfigur und die durchaus mögliche, ja von einer Aussage des achtzigjährigen Schriftstellers ${ }^{14}$ sogar angedeu-

8. - Siehe Jochen Eigler, „Krankheit und Sterben. Aspekte der Medizin in Erzählungen, persönlichen Begegnungen und essayistischen Texten Thomas Manns“, in: T. Sprecher, Liebe und Tod, a.a.O., S. 96-124, hier S. 106-111.

9. - Meldung vom 15. August $1891 \mathrm{im}$ Senatarchiv Lübeck, zitiert nach J. Eigler, ebd.

10. - Thomas Mann, Tagebücher 1918-1921, hrsg. von Peter de Mendelssohn, Frankfurt a.M., Fischer, 1979, S. 250 (25.5.1919).

11. - Thomas Mann, Gesammelte Werke in dreizehn Bänden, Frankfurt a.M., Fischer, 1974, Bd. XI, S. 536.

12. - H. Kurzke, Thomas Mann, a.a.O., S. 70.

13. - Ebd.

14. - In der oben erwähnten Ansprache in Lübeck sagte er 1955: „[Ich wünschte 
tete Last eines unbewussten Schuldgefühls gegenüber dem allzu früh verstorbenen Vater einzugehen, kann und muss die offensichtliche und allzu selbstverständliche Gewichtigkeit eines solchen Erlebnisses an der Schwelle zum Mannesalter hervorgehoben werden.

Nicht ohne Grund, und bestimmt nicht nur wegen einer in der damaligen Dekadenz modischen, von Schopenhauer abgeleiteten Todessymbolik, wimmelt es in Buddenbrooks von Todesszenen. Obwohl seine Biographie heute vielfach überholt ist, hat Roman Karst recht, wenn er auf die im Verlauf der Familienchronik immer ausgedehnteren Schilderungen der Todesfälle hinweist:

Der Todesschatten, der sich in vielen Details symbolisch manifestiert - im Schimmelgeruch, im dicken Strich unter der letzten Eintragung in der Familienchronik, im zugezogenen Vorhang -, begleitet den Knaben vom Tage seiner Geburt an. Hanno atmet den „fremden und doch so seltsam vertrauten Duft“" am Bett der sterbenden Großmutter, dann beim Leichnam des Vaters, und als der Tod sich schließlich auch bei ihm meldet, empfängt er ihn mit Erleichterung und Hingabe ${ }^{15}$.

Vor allem der Tod Senator Thomas Buddenbrooks, der sich genauso wie Senator Thomas Mann vergebens zur Erholung in Travemünde aufgehalten hat, zeugt von einer genauen Kenntnis des Sterbevorgangs und von dessen Wirkung auf den anwesenden Sohn:

Der kleine Johann saß Stunde für Stunde auf seinem Puff, sah alles an und horchte auf die gurgelnden Laute. Er hätte sich eigentlich zum Privatunterricht im Rechnen begeben müssen, aber er begriff, dass dies Ereignisse waren, vor denen die Kammgarnröcke verstummen mussten. Auch seiner Schulaufgaben gedachte er nur kurz und mit Spott... Manchmal, wenn Frau Permanender zu ihm trat und ihn an sich presste, vergoss er Tränen; aber meistens blinzelte er trockenen Auges mit einem abgestoßenen und grüblerischen Gesichtsausdruck darein, unregelmäßig und vorsichtig atmend, als erwarte er den Duft, den fremden und doch so seltsam vertrauten Duft...16

mir...], er hätte wenigstens meinen Weg noch etwas weiter verfolgen und sehen können, dass ich mich eben doch, gegen alles Erwarten, auf meine Art als sein Sohn, sein echter erweisen konnte“, in: T. Mann, Gesammelte Werke in dreizehn Bänden, a.a.O., Bd. XI, S. 535.

15. - Roman Karst, Thomas Mann. Eine Biographie, Kreuzlingen/München, Hugendubel (Focus Edition 12), 2006, S. 48.

16. - T. Mann, Buddenbrooks. Verfall einer Familie. Roman, hrsg. und textkritisch durchgelesen von Eckhard Hefrich, unter Mitarbeit von Stephan Stachorski und Herbert Lehnert, Frankfurt a.M., Fischer (Große kommentierte Frankfurter Ausgabe, Bd. I.1), 2002, S. 752-753. 
Natürlich darf man nicht versucht sein, die im Roman beschriebenen Szenen und Details als unmittelbare Verwendung realen Geschehens und Gefühls zu verstehen oder sie umgekehrt im Maßstab eins zu eins auf die Existenz des Schriftstellers zu projizieren: Thomas Mann ist nicht wie die Hauptfigur im Roman mit fünfzehn gestorben, aber er war (in seiner Vorstellung zumindest) fünfzehn, als er des Vaters beraubt wurde. Es ist wohl ein Teil von ihm in jenem Jahr, an jenem Tag gestorben. Erstaunlich und paradox wirkt es deswegen, wenn heute noch ein Brief vom 29. Dezember 1917 gern wortwörtlich gelesen wird, in dem Thomas Mann schreibt: „Ich war ein leichtsinniger Junge, als ich meinen Vater verlor, wohl impressionabel, aber doch eigentlich noch unfähig, den Tod geistig zu erleben [...]."17 Diese einzigartige Aussage wird bisher einer Relativierung des Trennungsschmerzes gleichgesetzt. In seinem umfangreichen Beitrag zu Krankheit und Sterben bei Thomas Mann beruft sich Jochen Eigler sogar auf einen emeritierten Ordinarius für Kinder- und Jugendpsychiatrie, um die Hypothese aufzustellen, dass ,ein 16jähriger junger Mann so mit sich selbst beschäftigt sein kann, dass ihm selbst der qualvolle Tod des Vaters kaum bewusst wird"18. Die Einschätzung ist an sich sicherlich korrekt (es ist nicht die Aufgabe des Literaturwissenschaftlers, im Bereich der Psychiatrie Urteile zu fällen), aber nichts, und vor allem nicht die besagte Stelle, erlaubt es, diese Diagnose auf den jungen Thomas Mann anzuwenden.

Wenn sich der Schriftsteller rückblickend einen leichtsinnigen und impressionablen Jungen nennt, heißt das nicht, dass ihm der Tod des Vaters gleichgültig geblieben sei. Ganz im Gegenteil: Mit „,impressionabel“ meint der erwachsene Thomas Mann, dass er „besonders empfänglich“ (Duden ${ }^{19}$ ), wenn nicht gar übertrieben reizbar gewesen sei. Die gewöhnliche Lektüre dieses zentralen Satzes beruht auf einem kompletten Missverständnis: Der Autor erklärt hiermit keineswegs, dass er damals wenig empfunden habe, sondern nur (man möchte sagen: sondern umgekehrt) dass er noch nicht in der Lage war, ,den Tod geistig zu erleben“. Geistig bedeutet mit Ruhe, mit Abstand, sachlich, trocken. Den Tod des Vaters hat Mann durchaus erlebt, sogar intensiv erlebt, aber eben nicht geistig. Diese Aussage zur Reaktion des sechzehnjährigen Waisen muss man unbedingt mit dem Brief vom 21. Juli 1897 in Verbindung bringen, in dem von „diskreten Formen und Masken“ die Rede ist. Manns Zurückhaltung in der Mitteilung der persön-

17. - T. Mann, Thomas Mann an Ernst Bertram. Briefe aus den Jahren 19101955, kommentiert und mit einem Nachwort versehen von Inge Jens, Pfullingen, Neske, 1960, S. 56 (29.12.1917).

18. - J. Eigler, „Krankheit und Sterben“, a.a.O., S. 112.

19. - http://www.duden.de/rechtschreibung/impressionabelhttp://www.duden.de/ rechtschreibung/impressionabel. Letzter Zugriff: 31.12.2016. 
lichsten Gefühle, seine prinzipielle Heimlichtuerei, die sich allerdings mit bloßem Totschweigen auch nicht zufriedengibt, seine Kunst der gezielten Verschlüsselung beschränkt sich nicht auf die homoerotische Problematik. Sie betrifft das gesamte Gebiet seiner tiefen Regungen. Thomas Mann verinnerlichte aufs Äußerste das Gebot der (patrizierhaften? bürgerlichen? norddeutschen?) Diskretion: Über Gefühle spricht man nicht. Mit Der kleine Herr Friedemann endet auf immer die Zeit der unreifen Larmoyanz. Wie Flaubert vor ihm kehrte er bewusst und entschieden der postromantischen Sentimentalität seiner jungen Jahre den Rücken.

Die auffällige Präsenz des Todes in den allerfrühesten Erzählungen erklärt sich also aller Wahrscheinlichkeit nach zu einem beträchtlichen Teil durch den schwer zu verkraftenden Verlust des Vaters. Übrigens dient in zwei dieser Erzählungen, die im Gegensatz zu den meisten anderen den Tod des Protagonisten als abschließenden Höhepunkt der Spannung nicht verwerten, nämlich Der Bajazzo und Tonio Kröger, der Tod des Vaters bezeichnenderweise als Wendepunkt im Schicksal der Hauptfigur. In der ersten steht eine extrem reduzierte Variation der Situation, die später in Buddenbrooks zur vollen Entfaltung kommen soll, ja eine Art Vorspiel, als würde der Schriftsteller sein Instrument im Kleinen üben. Der Ich-Erzähler, Sohn eines angesehenen Kaufmanns und einer künstlerisch begabten Mutter, wird von der Verpflichtung des Erbes insofern befreit, als sein Vater an dem Untergang des vier Generationen alten Familienunternehmens erkrankt und bald stirbt: „Eines Tages fand man ihn gelblich, gelähmt und lallend in dem Armsessel seines Privatcomptoirs, und eine Woche darauf nahm die ganze Stadt an seinem Begräbnis teil." 20 In dieser Version des Familienverfalls stirbt nicht der Sohn, sondern die Mutter, die nach einem halben Jahr ihrem Ehemann ins Grab folgt, während der lebensuntüchtige Protagonist wider Willen die traurige Existenz einer wandelnden Leiche weiterführt.

In der zweiten der beiden Erzählungen, welche während der Arbeit an den Buddenbrooks entstand, taucht erneut das Motiv der Sterbeszene anlässlich eines Besuches des ehemaligen, inzwischen zu einer Volksbibliothek umfunktionierten Familienhauses durch den seit vielen Jahren verreisten Sohn in komprimierter und durch ihre Sachlichkeit besonders packender Form auf:

20. - T. Mann, Der Bajazzo, in: Ders., Frühe Erzählungen 1893-1912. In der Fassung der Großen kommentierten Frankfurter Ausgabe, Frankfurt a.M., Fischer (Fischer Klassik), 2012, S. 131. Im Folgenden abgekürzt durch die Sigle FE und Seitenangaben in Klammern im Text. 
Das dort hatte als Schlafzimmer gedient. Seines Vaters Mutter war dort gestorben, so alt sie war, unter schweren Kämpfen, denn sie war eine genußfrohe Weltdame und hing am Leben. Und später hatte dort sein Vater selbst den letzten Seufzer gethan, der lange, korrekte, ein wenig wehmütige und nachdenkliche Herr mit der Feldblume im Knopfloch... Tonio hatte am Fußende seines Sterbebettes gesessen, mit heißen Augen, ehrlich und gänzlich hingegeben an ein stummes und starkes Gefühl, an Liebe und Schmerz. (FE, 290)

Wenn die Kurzform nicht dieselbe Ausführlichkeit ermöglicht wie die epische Breite einer (in der Taschenbuchausgabe) fünfhundert Seiten langen Familienchronik, bleibt die Häufigkeit des besonderen Leid-Motivs bis 1903 ein sicheres Zeichen ihrer Bedeutung für den jungen Schriftsteller. In den ersten Jahren seiner Schaffenszeit beschäftigt sich der junge Autor mehrmals literarisch mit der Erinnerung an die Todeskämpfe seiner Kindheit und insbesondere mit der Agonie seines Vaters, lernt den Tod ,geistig“ zu erleben. Schließlich geht es darum, eine Sprache zum Ausdruck einer der ergreifendsten Erfahrungen im Leben eines Menschen und eine Antwort auf die Frage nach dem Sinn des Daseins zu finden. „Geistig erleben“ bedeutet verarbeiten, überdenken, akzeptieren. Starke Gefühle, Liebe und Schmerz bleiben stumm; um sie auszusprechen, muss sie der Autor erst in Gedanken fassen.

Nach 1903, nach zehn Jahren Trauerarbeit also, rückt diese Thematik in den Hintergrund, bis sie im Tod in Venedig ihre unverminderte Aktualität unter Beweis stellt. Dort wird die infolge einer tiefen Schaffenskrise unternommene Reise des erfolgreichen, zu Lebzeiten sozusagen schon zum Klassiker gekrönten Schriftstellers Gustav von Aschenbach, der Züge des am 18. Mai 1911 gestorbenen Gustav Mahler trägt, zum Untergang in den Tod. In dieser mythisch-mystischen „Novelle“ greift Mann auf die uralte Tradition der Höllenfahrten zurück, wie Andrea Bartl erklärt:

Seine Reise nach und durch Venedig wird als Unterweltfahrt geschildert. An allen Schlüsselstellen, an denen die Handlung eine neue Wendung nimmt, trifft Aschenbach auf Charon-Gestalten, Konfigurationen des Todes: der fremde Reisende vor dem Münchner Nordfriedhof, der geschwätzige Schiffsoffizier, der Gondoliere, der Straßensänger auf dem Lido und Tadzio, der als Hermes psychagogos/ Hermes psychopompos Aschenbachs Seele am Ende der Novelle in die Unterwelt führen wird. Sie fungieren für den Leser, aber im Prinzip auch für Aschenbach selbst, als Vorausdeutungen: Der Zielpunkt seiner Reise wird der Tod sein ${ }^{21}$.

21. - Andrea Bartl, „Von geschminkten Greisen und schwarzen Schwänen“, in: Markwart Herzog (Hrsg.), Höllen-Fahrten. Geschichte und Aktualität eines Mythos, Stuttgart, Kohlhammer, 2006, S. 151-166, hier S. 162. 
Dabei sind die geschilderten Vorkommnisse erstaunlich wirklichkeitsgetreu. Als Thomas und seine Frau Katia gegen den 20. Mai 1911 in Venedig eintrafen, hatte nämlich eine Choleraepidemie die Lagunenstadt erreicht: Eine Wäscherin, die am Vortag in einer Kaserne gearbeitet hatte, brach am 22. Mai auf offener Straße zusammen; bereits am darauffolgenden Tag starb das erste Opfer der Seuche, und bis zum 16. Juni wurden 52 Fälle festgestellt, darunter 16 mit tödlichem Ausgang. Bis zum Ende der Epidemie am 2. November 1911 fielen insgesamt 88 Menschen der Seuche anheim. Der Titel der Erzählung, in der Thomas Mann über diese Erfahrung berichtete, ist also zunächst wortwörtlich zu verstehen: Während seines Aufenthaltes grassierte sehr wohl der Tod in Venedig.

In seiner außerordentlich minutiösen, geradezu unverzichtbaren Spurensuche hat Thomas Rütten überzeugend dargelegt, mit welcher Genauigkeit und Faktennähe, mit welchem ,,meisterlichen Realismus“22 der Schriftsteller trotz leichter Übertreibung bei der angegebenen Zahl der Todesfälle (dreißig in einer Woche, behauptet er) eine der letzten Choleraepidemien Europas geschildert hat. So gefährlich und unverantwortlich es auch klingen mag, versuchten die Behörden aus politischen und wirtschaftlichen Gründen tatsächlich, das Ausmaß der Seuche herunterzuspielen, die Presse mundtot zu machen und die Bevölkerung unter drakonischer Strafandrohung zum Schweigen zu bringen. Der Tod des Österreichers Anton Franzky, der nach einer Venedigreise in seiner Heimatstadt nahe Graz der Cholera asiatica erlegen war, machte tatsächlich den ganzen Juni in deutschsprachigen Zeitungen Schlagzeilen. Die Thomas Cook Reisegesellschaft, deren Niederlassung an der Piazzetta dei Leoncini Katia und Thomas Mann am 1. Juni tatsächlich besuchten, unterließ vom 20. Mai bis zum 12. August 1911 Reisen nach Italien. Deshalb darf man nach Rüttens Ansicht der Aussage des Schriftstellers Glauben schenken, wenn er 1930 in seinem Lebensabriss schrieb:

Ganz ebenso ist im Tod in Venedig nichts erfunden: Der Wanderer am Münchener Nordfriedhof, das düstere Polesaner Schiff, der greise Geck, der verdächtige Gondolier, Tadzio und die Seinen, die durch Gepäckverwechslung missglückte Abreise, die Cholera, der ehrliche Clerc im Reisebureau, der bösartige Bänkelsänger oder was sonst anzuführen wäre - alles war gegeben, war eigentlich nur einzustellen und erwies dabei aufs verwunderlichste seine kompositionelle Deutungsfähigkeit ${ }^{23}$.

22. - Thomas Rütten, „Die Cholera und Thomas Manns Der Tod in Venedig“, in: T. Sprecher (Hrsg.), Liebe und Tod, a.a.O., S. 125-170.

23. - T. Mann, „Lebensabriss“, in: Ders., Gesammelte Werke in dreizehn Bänden, a.a.O., Bd. XI, S. 98-144, hier S. 124. 
Aber nicht nur bei den Ereignissen hat sich Mann an die Wirklichkeit gehalten, sondern auch bei der Beschreibung der Seuche. Genauso wie bekanntermaßen im vorletzten Kapitel der Buddenbrooks der Todeskampf des fünfzehnjährigen Hanno dem Typhus-Artikel im Meyers Konversationslexikon von 1897 nachgedichtet ist ${ }^{24}$, wodurch der Tod des Protagonisten nicht leichtsinnig, emotional, pathetisch, sondern „geistig“ erlebt wird, bringt der Autor im fünften Kapitel des Tod in Venedig eine furchteinflößende Darstellung der Cholera, die nicht wenig der 14. Auflage des Brockhaus' Konversationslexikon ${ }^{25} \mathrm{zu}$ verdanken scheint, wie folgende Gegenüberstellung zeigt:

Seit mehreren Jahren schon hatte die indische Cholera eine verstärkte Neigung zur Ausbreitung und Wanderung an den Tag gelegt. Erzeugt aus den warmen Morästen des GangesDeltas, aufgestiegen mit dem mephitischen Odem jener üppig-untauglichen, von Menschen gemiedenen Urwelt- und Inselwildnis, in deren Bambusdickichten der Tiger kauert, hatte die Seuche in ganz Hindustan andauernd und ungewöhnlich heftig gewütet, hatte östlich nach China, westlich nach Afghanistan und Persien übergegriffen und, den Hauptstraßen des Karawanenverkehrs folgend, ihre Schrecken bis Astrachan, ja selbst bis Moskau getragen.

Aber während Europa zitterte, das Gespenst möchte von dort aus und zu Lande seinen Einzug halten, war es, von syrischen Kauffahrern übers Meer verschleppt, fast gleichzeitig in mehreren Mittelmeerhäfen aufgetaucht,
Die Cholera ist seit alter Zeit in gewissen Teilen Ostindiens (Niederbengalen, Malabarküste) heimisch, doch erst seit 1817 zeigt sie eine auffallende Neigung zur Ausbreitung und Wanderung. Nachdem sich bereits 1816 an den Gangesmündungen zerstreute kleinere Choleraherde gebildet hatten, dehnte sich die Krankheit im folgenden Jahre über die ganze Halbinsel aus, hatte am Schluss des J. 1818 bereits ganz Ostindien durchwandert, [...] verbreitete sich 1820-21 über ganz China und drang über Persien nach Astrachan. Ausgehend von einer neuen Epidemie, die 1826 in Bengalen ausgebrochen war, erreichte die Cholera 1829 von neuem die Ufer der Wolga, trat 1830 in Astrachan und zwei Monate später in Moskau auf. [...]

Während nämlich sonst die Krankheit [...] mehr als ein Jahr brauchte, ehe sie die europ. Grenze erreichte, gelangte sie diesmal in nur wenigen Tagen auf dem Seeweg von der Küste Arabiens aus nach Südeuropa [...].

Eine weitere Cholera-Epidemie brach, durch franz. Schiffe von Indien einge-

24. - Siehe T. Mann, Buddenbrooks..., a.a.O., S. 414 und S. 670-682.

25. - Brockhaus' Konversationslexikon, Leipzig/ Berlin/Wien, Brockhaus, 1902-1910, Bd. 4, S. 255, zitiert nach http://www.eLexikon.ch/54_0257. Letzter Zugriff: 31.12.2016. Vgl. auch Thomas Manns Arbeitsnotizen zur "Cholera asiatica“, in : T. Mann, Frühe Erzählungen. 1893-1912, Kommentar von Terence J. Reed unter Mitarbeit von Malte Herwig, Frankfurt a.M., Fischer (Große kommentierte Frankfurter Ausgabe, Bd. 2.2), 2004, S. 486-491. 


\begin{tabular}{|c|c|}
\hline $\begin{array}{l}\text { hatte in Toulon und Malaga sein Haupt } \\
\text { erhoben, in Palermo und Neapel mehr- } \\
\text { fach seine Maske gezeigt und schien aus } \\
\text { ganz Kalabrien und Apulien nicht mehr } \\
\text { weichen zu wollen. [...] [Das Übel] zeigte } \\
\text { häufig jene gefährlichste Form, welche } \\
\text { „die trockene“ benannt ist. } \\
\text { Hierbei vermochte der Körper das aus } \\
\text { den Blutgefäßen massenhaft abgeson- } \\
\text { derte Wasser nicht einmal auszutreiben. } \\
\text { Binnen wenigen Stunden verdorrte der } \\
\text { Kranke } \\
\text { und erstickte am pechartig zähe gewor- } \\
\text { denen Blut unter Krämpfen und heiseren } \\
\text { Klagen. (FE, } 578 \text { f.) }\end{array}$ & $\begin{array}{l}\text { schleppt, } 1884 \text { in Toulon und Marseille } \\
\text { aus, dehnte sich von da nach Italien, } \\
\text { besonders Neapel aus und suchte } 1885 \\
\text { Spanien heim. [...] } \\
\text { Bei der sog. trockenen Cholera (Cholera } \\
\text { sicca), einer besonders gefährlichen } \\
\text { Form, die aber selten auftritt, fehlen die } \\
\text { reiswasserähnlichen Ausleerungen gänz- } \\
\text { lich, weil der zeitig gelähmte Darmkanal } \\
\text { die in ihm ausgeschwitzten Stoffe nicht } \\
\text { auszutreiben vermag. Mit dem Eintritt } \\
\text { der wässerigen Ausleerungen stellt sich } \\
\text { ein quälender Durst [...] und der Tod } \\
\text { erfolgt gewöhnlich unter dem Zeichen } \\
\text { eines allgemeinen Blutstillstandes und } \\
\text { einer Nervenlähmung (Asphyktische } \\
\text { Cholera). [...] Das Blut ist dunkelblaurot, } \\
\text { mehr oder weniger eingedickt, in den } \\
\text { höhern Graden fast leer oder pechartig } \\
\text { zähe. }\end{array}$ \\
\hline
\end{tabular}

Die Montage hört allerdings nicht beim Brockhaus' Konversationslexikon auf, denn Mann hat sich offenbar, wie Laura Ottis vor einiger Zeit bemerkte, auch stark an der Beschreibung der Cholera in einer Konferenz des Mikrobiologen Robert Koch orientiert: „When Mann outlines the ways that cholera spreads, his notes follow the logic of Koch's article so closely that they might well have been taken from his original study.“ ${ }^{26}$ Die Konfrontation beider Texte bezeugt in der Tat an mancher Stelle eine gewisse Ähnlichkeit, vor allem in Bezug auf das Tigermotiv:

Erzeugt aus den warmen Morästen des Ganges-Deltas, aufgestiegen mit dem mephitischen Odem jener üppiguntauglichen, von Menschen gemiedenen Urwelt- und Inselwildnis, in deren Bambusdickichten der Tiger kauert, hatte die Seuche in ganz Hindustan andauernd und ungewöhnlich heftig gewütet $[\ldots] .(F E, 578)$

In diesem ganzen Gebiet an den Ufern des Ganges aufwärts bis Benares herrscht die Cholera beständig. [...] Eine üppige Vegetation und ein reiches Tierleben hat sich in diesem unbewohnten Landstrich entwickelt, der für den Menschen nicht allein wegen der Überschwemmungen und wegen der zahlreichen Tiger unzugänglich ist $[\ldots]^{27}$.

26. - Laura Otis, Membranes. Metaphors of Invasion in Nineteenth-Century Literature, Science, and Politics, Baltimore/London, The Johns Hopkins University Press, 1999, S. 158.

27. - Robert Koch, ,Erste Konferenz zur Erörterung der Cholerafrage am 26. Juli 1884 in Berlin“, S. 44, zitiert nach: http://edoc.rki.de/documents/rk/509-20-60/PDF/2060.pdf. Letzter Zugriff: 02.01.2016. 
Manns Schreiben über den Tod vermeidet jegliches Pathos zugunsten einer für den damaligen Stand der Medizin durchaus korrekten Sachlichkeit, deren vorgespielte Gefühlskälte weitgehend dem berühmten Prinzip des höheren Abschreibens entspringt. Die technische Sprache, die er hier nachahmt, gehört zu den Verschlüsselungsverfahren, durch die er seine Selbstbeherrschung zu bewahren versucht, zu den „novellistischen, öffentlichkeitsfähigen Formen und Masken“, die ihm erst die Mitteilung der geheimsten Empfindungen ermöglichen. Denn es ist kaum daran zu zweifeln, dass sich die Reaktivierung des Todesmotivs in der Venedig-Erzählung nicht allein durch die Erfahrung der Choleraseuche erklären lässt. Eine viel tiefergreifende Erfahrung hatte einige Monate vor seiner Ankunft in Venedig den Autor erschüttert, nämlich der Freitod der jüngsten Schwester am 30. Juli 1910.

Carla Mann war eine mittelmäßig begabte, erfolglose Schauspielerin, deren Hoffnung auf eine reiche, rettende Ehe zerbrach, als ihr Liebhaber einen anonymen Brief erhielt und seine Verlobte zur Rede stellte. Nach dem Gespräch kam die junge Frau nach Hause, schloss sich in ihrem Zimmer ein und erlebte einen furchtbar schmerzvollen Tod durch Zyankali. Bevor er ihr Schicksal im 35. Kapitel des Doktor Faustus (1947) einarbeitete, hatte Thomas Mann die furchtbare Szene schon 1930 in seinem Lebensabriss geschildert. Dabei fällt auf, wie die auf den Aussagen der Mutter beruhende Todesszene an den quälenden Durst und die Erstickung der Cholerakranken wenn nicht im Wortlaut, so doch im Duktus erinnert:

\begin{tabular}{|l|l|}
\hline Binnen weniger Stunden verdorrte der & {$[\ldots]$ das letzte, was von ihr laut wird, } \\
Kranke und erstickte am pechartig zähe & ist das Wassergurgeln, womit sie die \\
gewordenen Blut unter Krämpfen und & Verätzungen in ihrem Schlunde zu \\
heiseren Klagen. Wohl ihm, wenn, was & kühlen sucht. [...] Dunkle Flecken an den \\
zuweilen geschah, der Ausbruch nach & Händen und im Gesicht zeugten von dem \\
leichtem Übelbefinden in Gestalt einer & Erstickungstod, der, nach einem kurzen \\
tiefen Ohnmacht erfolgte, aus der er nicht & Zögern der Wirkung, jäh gewesen sein \\
mehroder kaum noch erwachte. $(F E, 579)$ & mochte ${ }^{28}$.
\end{tabular}

Weit davon entfernt, die Präsenz der toten Schwester im Tod in Venedig zu widerlegen, verstärkt die Nähe dieser Textstelle zum Artikel „Cholera“ im Brockhaus' Konversationslexikon ganz im Gegenteil die Vermutung eines direkten Zusammenhangs mit dem traumatisierenden Erlebnis der freiwilligen Vergiftung der Schwester, da die so eindrucksvolle Bezeichnung des ,pechartig zähen“ Blutes bei der Leichenöffnung in der Enzyklopädie mitnichten auf Erstickung zurückgeführt wird. Hier scheint der persönliche Bezug des Autors zur Todesthematik durchzu-

28. - T. Mann, „Lebensabriss“, a.a.O., S. 120-121. 
blicken: Die verzweifelte Geste der erpressten Untreuen bestürzte jedes Familienmitglied auf eine jeweils andere Art und Weise, für Thomas war sie nach eigener Aussage ,wie ein Verrat an [der] geschwisterlichen Gemeinschaft" ${ }^{29}$. Seinem älteren Bruder schrieb er am 4. August 1910: „Mein geschwisterliches Solidaritätsgefühl lässt es mir so erscheinen, da $\beta$ durch Carlas Tat unsere Existenz mit in Frage gestellt, unsere Verankerung gelockert ist." ${ }^{30}$ Ganz wahrscheinlich öffnete sich also neu eine verheilt geglaubte Wunde. Der Selbstmord der Schwester zerstörte die nach dem Tod des Vaters mit Mühe erfundene Legende des Familienzusammenhalts. Auch davon handelt, vielleicht sogar vorrangig, Der Tod in Venedig.

Es wäre jedenfalls verfehlt, die Todesfälle und -szenen in Manns Frühwerk auf eine zeitgeistbedingte Metapher oder einen erzählerischen Kniff zu reduzieren. Sicherlich bildet der Tod des Protagonisten in mehreren Erzählungen ein spannungserzeugendes Mittel: Sowohl in Der kleine Herr Friedemann als auch im Tod in Venedig ist die allerdings schon von Tolstoi am Ende seiner 1886 veröffentlichten Erzählung Der Tod des Iwan Iljitsch experimentierte Innenansicht des letzten Augenblickes ein zeittypischer Kunstgriff, mit dem der Endpunkt zum befürchteten Höhepunkt wird. Sicherlich nimmt die Erfahrung des Todes in Manns hochraffiniertem Erzählgebilde einen symbolischen bzw. mythischen Wert ein, dennoch resultiert dieser aus einem tatsächlichen Erlebnis des Todes, das der Autor in seinem Werk zu einem geistigen Erlebnis umwandelt. Mann bemüht sich, dem unbegreiflichen Phänomen des Todes einen Sinn abzugewinnen. Er beschränkt sich nicht auf die Schilderung des körperlichen Todes, sondern ordnet ihn in eine allgemeine Lebensauffassung ein. Nicht von ungefähr schreibt er 1930 in seinem Lebensabriss: „Alle Wirklichkeit hat todernsten Charakter"31.

Während unter „Leben“ ein gesundes Leben zu verstehen ist, etwa die fröhliche, unbekümmerte Existenz des Hundes Esau in Tobias Mindernickel, des Großkaufmanns Klöterjahn in Tristan, des vergötterten Hans Hansen in Tonio Kröger oder der russischen Familie im Tod in Venedig, um einige Beispiele aus den berühmtesten Erzählungen heranzuziehen, wird Tod zu einem Synonym oder Sinnbild für ein unzufriedenes, krankhaftes, gequältes Leben. Das Hinscheiden zahlreicher Hauptfiguren ist die erzähltechnisch logische Folge einer grundsätzlichen Lebenseinstellung, eines unmöglichen unbekümmerten Daseins, einer schwierigen, vom Gedanken an den Tod bedrohten Existenz. Es

29. - Ebd.

30. - Thomas Mann/Heinrich Mann, Briefwechsel. 1900-1949, hrsg. von Hans Wysling, Frankfurt a.M., Fischer, 1984, S. 111 (4.08.1910).

31. - T. Mann, „Lebensabriss“, a.a.O., S. 121 (kursiv vom Autor). 
veranschaulicht nur eine längst vor dem letzten Atemzug vorhandene Unfähigkeit zur inneren Ruhe.

Die Lust auf ein Künstlerleben, die Liebe für Literatur und Musik, die Abneigung gegen Leibesübungen und praktisches Denken ging zweifelsohne dem Tod des Vaters voraus, aber erst nach diesem Verlust entwickelte der junge Thomas Mann ein Interpretationssystem, in dem Geschäft für Leben steht und Kunst unabwendbar mit dem Tod zusammenhängt, erst danach kam er zur Überzeugung, ,daß gute Werke nur unter dem Druck eines schlimmen Lebens entstehen, daß, wer lebt, nicht arbeitet, und daß man gestorben sein muß, um ganz ein Schaffender zu sein" (Tonio Kröger, $F E$, 266). Durch dieses alles andere als selbstverständliche Postulat versuchte er mit den Widrigkeiten der eigenen Existenz eher schlecht als recht zurechtzukommen. Auf die niederschmetternde Auseinandersetzung mit dem Tod reagiert er stets kühl, selbstbeherrscht, mit einer gewollten Ruhe. Die verdrängten Gefühle kommen aber verklärt zum Ausdruck, d.h. verschlüsselt, geistig geformt, ins Ästhetische übertragen. Es ist eine der bewundernswerten Leistungen des Schriftstellers, dass die Motive und Situationen in seinem Werk nicht bloße Allegorien sind, die abstrakte Inhalte oder persönliche Belange sinnbildlich darstellen, sondern sich die Übereinstimmungen in beliebiger Richtung verfolgen lassen, ohne dass eine Bedeutung die anderen überragt. Es sind nicht einfach Masken, die es herunterzureißen reichen würde, um dahinter eine unaussprechliche Wahrheit zu entdecken, es sind gegenseitige Entsprechungen in einer höchstkomplizierten Äquivalenzrelation.

Aschenbach muss nicht nur deswegen sterben, weil die Cholera 1911 in Venedig wütet, oder nicht nur deswegen, weil seine verwerfliche, pädophile Liebe für einen „Knaben“ sein bisheriges geregeltes Leben zerstört, weil die Entdeckung der wahren Schönheit seine Kunstauffassung und sein bürgerlich anerkanntes Werk zugrunde richtet, weil ihn ein Charonähnlicher Gondoliere über die Acheron gleiche Laguna auf dem elysischen Lido absetzt, wo ihn ein fremder Gott in den Tod verführt, weil sich Thomas Mann nach dem Selbstmord seiner Schwester mit dem Tod auseinandersetzen musste, sondern aus all diesen Gründen zugleich und gleichrangig. Mit anderen Worten: Der Tod in Manns Frühwerk reduziert sich nicht auf eine Chiffre, die auf andere Dinge oder Begriffe metaphorisch verweisen würde, er bezieht sich auch auf den Tod an sich, auf die Frage nach dem Sinn des Lebens nach der Zerstörung der heiligen Kernfamilie, auf den unerträglichen, unsagbaren Schmerz nach dem Tod eines nahen, geliebten Verwandten. Der Umweg über medizinische, kunstphilosophische oder mythische Darstellungen des Todes gehört zu Manns Strategie der erzählerischen Verschlüsselung. In seiner Lebensauffassung hängt die Kunst unmittelbar mit dem Tod zusammen, denn die Unsterblichkeit der wahren Kunst ist in seinen Augen die einzige Rettung vor dem sinnzerstörenden Tod. 